\title{
Synergistic Effect and Formulation Optimization of Antioxidant between Tea polyphenols and sorghum red pigment
}

\author{
Yangbiao Zhang ${ }^{\mathrm{a}}$,Xiaolei Chen ${ }^{\mathrm{b}}$, Zhiyou Zhic, Yihua Jiang ${ }^{\mathrm{d}, *}$ \\ ${ }^{1}$ Zhejiang Shuren University, Hangzhou, China

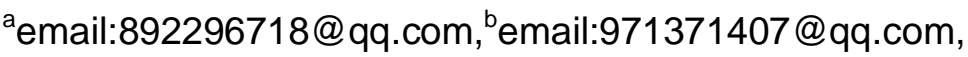 \\ cemail:592140367@qq.com, ${ }^{\mathrm{d}}$ email:hzjyih828@163.com
}

\begin{abstract}
Keywords:antioxidant;synergistic effects; tea polyphenols;sorghum red;response surface method(RSM).

Abstract: In order to understand the synergetic effect between the solution of tea polyphenols and sorghum red pigment, synergetic effect and optimal formulation of compound antioxidant with the response surface method were determined using the method of free radical scavenging under DPPH system. The results showed that, the component ratio of optimal compound antioxidant was tea polyphenols $6.00 \mu \mathrm{g} \cdot \mathrm{ml}^{-1}$ and sorghum red $293.76 \mu \mathrm{g} \cdot \mathrm{ml}^{-1}$ Under these conditions, the DPPH scavenging rate of the system was $54.96 \%$, compound antioxidant of tea polyphenols and sorghum red had antagonistic action. Through the comparison of optimal mixed antioxidants and antioxidant effect of single antioxidant,we concluded that mixed antioxidants were poorer than single antioxidant.
\end{abstract}

\section{Introduction}

Tea polyphenols (TP) is one of the main pharmacological ingredient tea and is a general term for the tea polyphenols.Tea polyphenols has strong antioxidant activity and free radical scavenging capacity[1]. There are synergistic antioxidant effect not only between the monomer polyphenols, but also between tea polyphenols and Vc, Ve [2-3]. The research results of Wang YF etc[4] also indicated that the synergistic effect of tea polyphenols and several kinds of natural antioxidants. The research is still blank whether there are synergistic effects and this synergistic effect is weakened or enhanced etc between tea polyphenols and anthocyanin.DPPH - method is used to evaluate a natural antioxidant activity with simple, rapid, sensitive and feasible [5-6]. The DPPH. scavenging rate as evaluation index of antioxidant activity,the sorghum red as a typical representative of anthocyanin pigment, the synergetic effect and optimal formulation between tea polyphenols and sorghum red were determined using the response surface optimization method under DPPH. scavenging system in this paper.

\section{Material and Methods}

\section{Materials, Reagents}

Tea polyphenols was prepared by our subject group with Longjing Green Tea , purity $>98 \%$. sorghum red pigment (content 41.32\%) was purchased from Qianjiang green sen treasure bio-tech Co., ltd.

$\mathrm{DPPH} \cdot$ was purchased from Sigma company, other reagents were of analytic grade. Distilled water was used throughout the experiment.

\section{Antioxidant test}

$\mathrm{DPPH} \cdot$ is a stable free radical, which has been widely used in phytomedicine for the assessment of scavenging activities of bioactive fractions.DPPH radical-scavenging activity was performed by the method described of Ying QL [7]. The stock solution of DPPH. was prepared by dissolving $25.63 \mathrm{mg}$ in $100 \mathrm{ml}$ absolute ethyl alcohol $\left(6.5 \times 10^{-4} \mathrm{~mol} / \mathrm{L}\right)$. For each determination, the stock solution was diluted to a dilution to $6.5 \times 10^{-5} \mathrm{~mol} / \mathrm{L}$ with $60 \%(\mathrm{v} / \mathrm{v})$ ethanol. An aliquot of each dilution $(1.0 \mathrm{~mL})$ was mixed with solution of DPPH $\left(5 \mathrm{~mL}, 6.5 \times 10^{-5} \mathrm{~mol} / \mathrm{L}\right)$. The mixtures were shaken vigorously and incubated at $37{ }^{\circ} \mathrm{C}$ in the dark for $30 \mathrm{~min}$. At the same time, a control containing $60 \%$ (v/v) ethanol 
$(1.0 \mathrm{~mL})$ and solution of DPPH $\left(5 \mathrm{~mL}, 6.5 \times 10^{-5} \mathrm{~mol} / \mathrm{L}\right)$ was run. The absorbance was measured at $517 \mathrm{~nm}$ against $60 \%(\mathrm{v} / \mathrm{v})$ ethanol $(5.0 \mathrm{~mL})$ mixed tested extract solution $(1.0 \mathrm{~mL})$ as a blank. The percentage of DPPH scavenging was calculated as follows: $\mathrm{P} \%=\left[1-(\mathrm{Ai}-\mathrm{Aj}) / \mathrm{A}_{0}\right] \times 100$, where $\mathrm{A}_{0}$ is absorption of control, $\mathrm{Ai}$ is absorption of tested extract solution, $\mathrm{Aj}$ is absorption of blank.

\section{Synergy analysis method}

The effect produced exceeds the sum of their respective alone when used in combination of several substances, the synergistic effect is produced.The synergy evaluation method of antioxidant in vitro have three methods of the addition method,the direct comparison method and the response surface method commonly. The plus method suitable for single component in large difference antioxidant activity,the direct comparison method is mainly suitable for the single component in such similar antioxidant activity under the same concentration.Response Surface Methodology (RSM) is a effective for responses that can reflect the experimental method of synergistic effect between each factor[8],it can optimize process parameters by combination of mathematics and statistics method to fit the factors and response value by quadratic regression equations.Comparison of the response coefficients in the equation, can be quantified analysis factors influence on the composite antioxidant activity from the view of mathematics [9] . The subject chosen response surface methodology (RSM) .

\section{Response surface test method}

\section{The single factor test}

The sample solution $(1 \mathrm{mg} / \mathrm{ml})$ was dissolved with water. The sample solution was mixed with DPPH.solution $\left(5 \mathrm{~mL}, 6.5 \times 10^{-5} \mathrm{~mol} / \mathrm{L}\right)$. To add a small amount the sample solution at first, increases gradually, and observe the fade of solution while still mixing. When the solution basic color faded, remembered the concentration of sample. The maximum concentration was this concentration.Based on the maximum concentration, set up the appropriate gradient, make arithmetic sequence.According to the antioxidant test method of single factor tested.

\section{The response surface experimental design}

According to the single factor experiment results, with the concentration of tea polyphenols, sorghum red as independent variables,response surface methodology experiments of Box-Behnken design with 2 factors and 3 levels were chosen for formulation optimization of tea polyphenols and sorghum red pigment. The factors and levels were listed in Table 1.

Table 1 Levels of factors in response surface methodology

\begin{tabular}{lccc}
\hline \multirow{2}{*}{ Factor } & \multicolumn{3}{c}{ Level } \\
\cline { 2 - 4 } A Tea polyphenols $\left[\mu \mathrm{g} \cdot \mathrm{ml}^{-1}\right]$ & 2 & 0 & 1 \\
\hline B sorghum red $\left[\mu \mathrm{g} \cdot \mathrm{ml}^{-1}\right]$ & 100 & 4 & 6 \\
\hline
\end{tabular}

\section{Result and analysis}

\section{The single factor test}

\section{The DPPH· scavenging rate of the tea polyphenols}

The experimental results were shown in figure 1 . Figure 1 showed, tea polyphenols had the effect of scavenging DPPH free radical, and had a high dose effect relationship.The antioxidant ability of tea polyphenols was strengthens gradually with the increase of concentration.Three levels were of $2,4,6 \mu \mathrm{g} / \mathrm{ml}$ were determined at tea polyphenols concentration.

\section{The DPPH· scavenging rate of the sorghum red}

Sorghum red pigment is isoflavones galactoside, a kind of natural pigment from the kaoliang shell and safety,not poisonous edible.Main components of red pigment were 5,4'-two hydroxyl isoflavones-7-O-galactoside and 5,4'-two hydroxyl-6,8-two methoxy isoflavone 
7-O-galactoside[10].It showed a higher scavenging activity against free radicals such as $\mathrm{DPPH} \cdot[11]$.The experimental results were shown in figure1. Figure 1 showed, the ability of scavenging DPPH increased with increasing concentration in the $800 \mu \mathrm{g} / \mathrm{ml}$ range of sample concentration. The change of the scavenging DPPH ability tended to be stable when the concentration was more than $800 \mu \mathrm{g} / \mathrm{ml}$.Three levels were of $100,200,300 \mu \mathrm{g} / \mathrm{ml}$ were determined at sorghum red concentration.

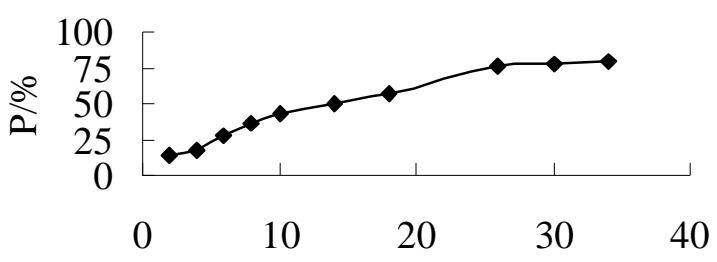

Tea polyphenols concentration/ $/ \mathrm{gg} \cdot \mathrm{ml}^{-1}$

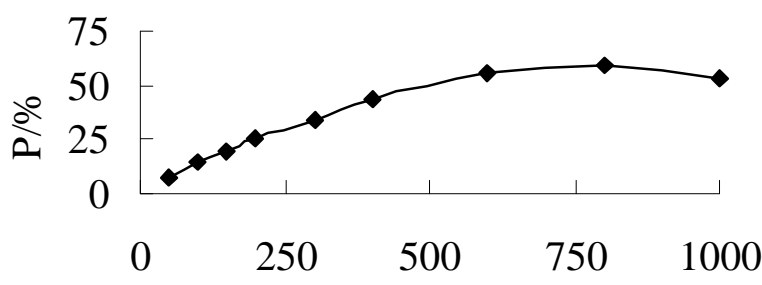

Sorghum red concentration $/ \mu \mathrm{g} \cdot \mathrm{ml}^{-1}$

Fig.1 Effects of different factors on the DPPH· scavenging rate

\section{The test results of RSM}

Based on the results of single factors experiments, the formulation optimization of Tea polyphenols and sorghum red pigment experiments were carried out by RSM design according to Table 2.

Table 2 Data of RSM

\begin{tabular}{cccc}
\hline No. & A & B & P ( \% ) \\
\hline 1 & 0 & 1 & 45.96 \\
2 & 1 & 1 & 54.12 \\
3 & 0 & 0 & 40.65 \\
4 & 0 & 0 & 46.33 \\
5 & 0 & 0 & 47.80 \\
6 & 0 & 0 & 39.01 \\
7 & -1 & 1 & 47.71 \\
8 & -1 & 0 & 34.76 \\
9 & 0 & 0 & 40.92 \\
10 & -1 & -1 & 26.76 \\
11 & 1 & 0 & 51.43 \\
12 & 0 & -1 & 24.20 \\
13 & 1 & -1 & 36.22 \\
\hline
\end{tabular}

\section{Analysis of variance}

Analysis of variance was carried out by design expert and the results showed that the p values of tea polyphenols (A) and sorghum red(B) were all less than 0.01 , which showed that the factors had most important influence on the scavenging DPPH. Analysis of variance showed that the factors and responses were not linear relationship. Above results showed that the specific factors and scavenging response did not have simple correlation relations. 
Table 3 Analyze of mean square

\begin{tabular}{cccccc}
\hline $\begin{array}{c}\text { Sourc of } \\
\text { Squares }\end{array}$ & Sum of Square & $\begin{array}{c}\text { Degree of } \\
\text { freedom }\end{array}$ & Mean Square & F Value & Prob > F \\
\hline Model & 1520.10 & 5 & 172.75 & 10.83 & 0.0034 \\
A & 563.96 & 1 & 176.48 & 11.06 & 0.0127 \\
B & 744.60 & 1 & 612.26 & 38.37 & 0.0004 \\
AB & 12.78 & 1 & 2.33 & 0.15 & 0.7140 \\
$\mathrm{~A}^{2}$ & 141.19 & 1 & 25.44 & 1.59 & 0.2472 \\
$\mathrm{~B}^{2}$ & 133.22 & 1 & 68.50 & 4.29 & 0.0770 \\
Residual & 205.80 & 7 & 15.96 & & \\
Lack of Fit & 163.08 & 3 & 17.28 & 1.15 & 0.4299 \\
Pure Error & 42.72 & 4 & 14.97 & & \\
Cor Total & 1725.90 & 12 & & & \\
\hline
\end{tabular}

\section{Fitting the model to establish and antioxidant synergy analysis}

To fit the response surface test data, to obtain the coding variable regression equation of two order polynomia. $\mathrm{P}=+42.12+5.42 \mathrm{~A}+10.10 \mathrm{~B}-0.76 \mathrm{~A} \mathrm{~B}+3.03 \mathrm{~A}^{2}-4.98 \mathrm{~B}^{2}$

The $p$ vale of the lack of fit value was of 0.4299 (not significant), the model was of 0.0034 (significant), which showed that the model fit the RSM data significantly, the equation was of the good mathematic model to fit the synergetic antioxidant effect between the tea polyphenols and sorghum red pigment.

According to the regression equation,the first degree were always positive, indicating that tea polyphenols and sorghum red were positive correlation with the scavenging rate of DPPH.,the interaction coefficient of tea polyphenols and sorghum red was negative value, reflecting the synergistic effect between two factors was negative cooperativity, composite antioxidant activity decreased with the increase of the antioxidants concentration.

Using the antioxidants concentration of tea polyphenols and sorghum red as $\mathrm{X}$ and $\mathrm{Y}$, as the $\mathrm{Z}$ in the cavenging rate of $\mathrm{DPPH} \cdot$, make the corresponding $3 \mathrm{D}$ figure. The interaction between tea polyphenols(A) and sorghum $\operatorname{red}(\mathrm{B})$ had great influence on the response, which showed relatively steep curve in the figures and it was consistent with the regression analysis results.

\section{The optimal process validation}

The equivalent linear regression model was shown in Figure 2.The maximum value of model was calculated by the software. The component ratio of optimal compound antioxidant was sorghum red $293.76 \mu \mathrm{g} \cdot \mathrm{ml}^{-1}$, tea polyphenols $6.00 \mu \mathrm{g} \cdot \mathrm{ml}^{-1}$, the DPPH scavenging rate was $54.96 \%$, the verified value could reach above $54.5 \%$, it was consistent with the predicted value and verified the validity of the model. The single component test with combination formulations of sorghum red $293.76 \mu \mathrm{g} \cdot \mathrm{ml}^{-1}$,tea polyphenols $6.00 \mu \mathrm{g} \cdot \mathrm{ml}^{-1}$, the actual production of DPPH $\cdot$ scavenging rate were: $31.30 \% \%, 27.80 \%$, the total scavenging rate of $59.10 \%$,respectively.It was slightly smaller than the combined formula.It showed that combined formula antioxidant activity was poorer than single antioxidant and consistent with the regression model analysis. 

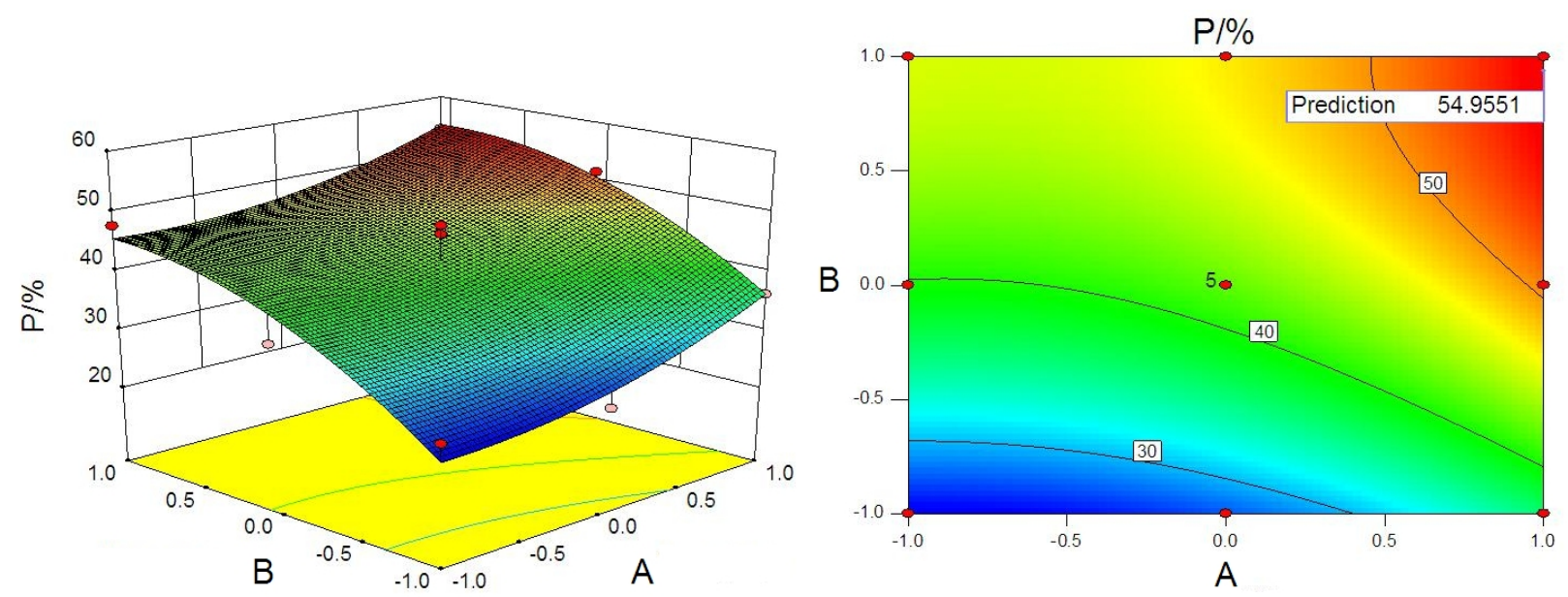

Fig.2 The equivalent linear regression model and $\mathrm{P}=\mathrm{f}(\mathrm{A}, \mathrm{B})$ response surface

\section{Conclusion}

In this study, in order to understand synergetic antioxidant effect between the tea polyphenols and sorghum red pigment, synergetic effect and optimal formulation of compound antioxidant were determined with the response surface method.The optimum formula of compound antioxidants were : tea polyphenols $6.00 \mathrm{~g} \cdot \mathrm{ml}^{-1}$ and sorghum red $293.76 \mathrm{~g} \cdot \mathrm{ml}^{-1}$, the DPPH. scavenging rate was $54.96 \%$ under the conditions. Comparison the antioxidant activity between compound antioxidants and single antioxidant, concluded that mixed antioxidants slightly poorer than single antioxidant. In the low concentration, there were antagonistic action of antioxidant, but the effect was not significant.

\section{Acknowledgments}

This work was supported by National innovation and entrepreneurship training program (No.201611842004) .

\section{References}

[1] Xu P,Liu D C,Study on the antioxidant and antibacterial mechanism of tea polyphenols[J].China herald of Medicine.2008,5(23):21-22.

[2] Zhao B L,The synergistic effects of polyphenols[J]. Chinese Science Bulletin, 2002,16 (47):1206-1210.

[3] Hu X F, Mao J M, Jiang L P, The synergistic effects of polyphenols and other antioxidants[J]. Tea, 2000,26(2):66-69.

[4] Wang Y F, Xu P, Li L, Study on synergistic effect of tea polyphenols and several kinds of natura antioxidants[J]. Tea Science, 2010,30(2):109-114.

[5] Takara K,Otsuka K,Wada K,et a1.1,1-Diphenyl-2-picrylhydrazyl radical scavenging activity and tyrosinase inhibitory effects of constituents of sugarcane molasses[J].Biosci Biotechnol Biochem,2007,71(1):183-191.

[6] Okawa M,Kinjo J,Nohara T,et a1.DPPH (1,1-dipheny-2-picrylhydrazy1)radical scavenging activity of flavonoids obtained from some medicinal plants[J].Biol Pharm Bull,2001,24(10):1202-1205. 
[7] Ying Q L,Study on debinding and antioxidant technology eel[D].Hzngzhou:Zhejiang Gongshang University.2011

[8] S.J. Kalil,F.Maugeri,M.I.Rodrigues. Response surface analysis and simulation as a tool for bioprocess design and optimization[J].Process biochemistry, 2000,35:539-550.

[9] Tan Z Q,Wu W L,Pan L Y,Research on Compound Antioxidants on low energy of cocoa butter equivalent antioxidant[J].Science and technology of food industry,2012,33(19):185-187,291.

[10] Wang $\mathrm{J}$ T,Research advances on natural red pigment form sorghum bicolor[J]. Cereals \& Oils,2012(11):7-11.

[11] Zhu Y H,Yang J X,Dai B,In vitro antioxidative properties of sorghum red pigment[J]. Journal of Shaanxi Normal University(Natural Science Edition),2009,37(3):66-69. 